\title{
DEVELOPMENT OF A 3D INFORMATION SYSTEM FOR THE OLD CITY CENTRE OF ATHENS
}

\author{
Nikos KASKAMPAS, Kalli SPIROU-SIOULA, Charalabos IOANNIDIS \\ National Technical University of Athens, School of Rural \& Surveying Engineering \\ 9 Iroon Polytechniou St, Athens 15780, Greece \\ nkaskampas@gmail.com; kallispyrou@gmail.com; cioannid@survey.ntua.gr
}

Keywords : 3-D City Modeling, GIS, Geometric Documentation, Visualization, Cultural Building

\begin{abstract}
The representation of three dimensional city models has been gaining ground increasingly in many scientific fields in the recent years. 3D City Modelling is a scale representation of natural and artificial objects in order to present the spatial data and highlight the social development of the city. Depending on its importance or the purpose of use, an object can be represented in various levels of detail. An increasing tendency to 3D city models is their integration into GIS, which proves to be an effective tool for managing, analyzing and planning in order to make decisions about technical, administrative and financial matters. A combination of digital photogrammetric techniques and laser scanning data contribute greatly to this, since a variety of data, such as aerial, satellite and terrestrial images, point clouds from airborne and terrestrial laser systems, and also a variety of photogrammetric and mobile mapping methods are available. The objective of this paper is the development of a 3D Information System (IS) for the three-dimensional geometric documentation of the buildings owned by the Ministry of Culture in the old city centre of Athens, Greece, named "Plaka". The area has been inhabited continuously since the prehistoric era, it has a special architectural style and includes a number of unique cultural heritage monuments. The data used for the reconstruction of the 3D model of Plaka consisted of aerial and terrestrial images, while raster, vector and descriptive data were used for the creation of a 2D GIS, which served as the background for the development of the 3D GIS. The latter includes all of the qualitative and quantitative information related to the 3D building models owned by the Ministry of Culture according to users' needs. Each building in the vicinity of Plaka was depicted in one of the four different levels of detail created for the purpose of the study, according to their ownership status and other criteria. The building models, depicted in the highest level of detail, were owned by the Greek Ministry of Culture whereas the other buildings (of a lower level of detail) were depicted in a more subtractive way. Therefor an integrated IS was developed that combines descriptive information, e.g., use, legal status, images, drawings, etc, with the spatial information and geometric documentation in three dimensions.
\end{abstract}

\section{INTRODUCTION}

Three dimensional (3D) City Modelling is a scale representation of natural and artificial objects intended to present the spatial data and highlight the social development of the city. The fast, accurate and reliable reconstruction of such a model provides a comprehensive view of the development and management of the city, which is essential to reduce risks and improve its effective management [1]. So the 3D city modelling has a continuously growing range of applications. The integration of a 3D model into a 3D Information System (IS) enables the management of data and processes related to a city's infrastructure. The major factors requiring the development of a 3D GIS for the threedimensional spatial and descriptive information management are the need for better visualization of objects, the restitution of their various attributes in 3D space so that semantically rich georeferenced 3D models may be created, and the possibility of better information management regardless of the types of data storage and operating systems used [2, 3]. These systems have the advantages of a 2D GIS while also provide visualization and navigation in the model space. They are used in many study areas such as ecological studies, environmental monitoring, geological analysis, mining exploration, architecture, automatic vehicle navigation, archaeology, 3D urban mapping and landscape planning. The integration of 3D city models in GIS not only provides the spatial information of depicted objects but also allows their relation to important information while maintaining the identity of the city, especially when it is of great historic significance. Therefore, this integrated system allows the monitoring of the status quo in order to take appropriate measures to safeguard the cultural heritage of buildings, and to intervene at any economic or architectural level [4]. The techniques used to model city objects include terrestrial surveying, aerial surveying and integration processes. The criteria to be considered are the level of detail and the accuracy, the accessibility of the buildings and of their specific characteristics, and the available data in the area. Photogrammetry and laser systems (LIDAR and mobile terrestrial laser scanners) constitute the main tools for geometrical documentation of objects. In many cases integrated Mobile 
Mapping Systems are used, consisting of a moving platform that includes navigation sensors and mapping sensors [5, 6]. Mapping sensors include pushbroom line scanners (2D time-of-flight laser scanners) and digital and video frame cameras of usually small format and large frequencies for image capturing $(7-15$ frames per sec $)$ with a small exposure time to avoid blur. Usually, camera systems with 6-8 CCD video cameras are used to provide terrestrial panoramic images. Automated procedures for 3D model creation are used for fast processing of multiple oblique terrestrial images in combination with terrestrially or aerially collected 3D point clouds [7]. The result is the creation of walk- or fly-through models or the panoramic views of the urban spaces (street views). Several examples of 3D real or augmented models of various cities all over the world are already available (e.g., for Berlin, Montreal, New York, Tokyo, Venice, Vienna, Paris, etc.) and can be found at web-sites such as www.3dcadbrowser.com, www.cybercity3d.com, www.computamaps.com, etc. In this paper the development of a 3D IS for the documentation of the buildings owned by the Ministry of Culture in Plaka, the old city centre of Athens, Greece, using aerial and terrestrial imagery and field measurements is described. Firstly a 2D GIS was developed after collecting all the necessary raster, vector and descriptive data, which served as the background for the creation of the 3D GIS. The latter includes all the qualitative and quantitative information related to the 3D building models owned by the Ministry of Culture, according to user's needs. The buildings were depicted in four different levels of detail according to specific criteria (e.g., ownership status, historical or architectural importance, etc). For the implementation of the study a variety of software were used. In particular, data acquisition and editing was achieved using AutoCad, Photoshop CS3, Archis and Stereo Soft Kit (photogrammetric packages), while 3D Studio Max and Google Sketchup 6.0 were used for the modeling of the buildings. Finally, ArcInfo 9.3 was used for the development of the 2D and 3D GIS. The integration of $3 \mathrm{D}$ city models in GIS enables the storage of important information so as to maintain the identity of the city, especially when it is of great historic significance.

\section{PLAKA: THE OLD CITY CENTRE OF ATHENS}

Plaka area is situated in the centre of Athens, Greece, at the foot of the hills where Acropolis was built. Since the 1830's when the name Plaka was given to the region, it has remained a part of the greater city of Athens over the centuries having its history bound up with that of Athens. Athens has been continuously inhabited since $3500 \mathrm{BC}$. Times of rise and decline succeeded one another making it a place of great changes and interest. A vast amount of monuments and ruins can be found scattered around Athens, each one reminding of a different historic era. Especially in Plaka one can see the Ancient Agora, Roman buildings, such as the Library of Hadrian and the Odeon of Herodes Atticus, churches of the Byzantine period and buildings constructed during the Turkish occupation. The layout of Plaka has undergone many changes over the centuries. As shown by the traces of walls, the size of the city was increasing or decreasing depending on the periods of prosperity at the time of war or peace. During the Archaic period at the time of Solon the city occupied a small area of 52 ha around the Acropolis which later increased to 250 ha during the Hellenistic period until the fall to the Franks when the city diminished. At the period of the Ottoman rule, the city was slowly beginning to grow and spread. It is the period from which the region has inherited its current layout. Today Plaka has become a touristic area. The buildings are homogenous since the majority of them were built at the request of the government in 1835 when intensive construction works took place in downtown Athens. However, despite the studies that were carried out, the layout of Plaka remained unchanged from the years of Ottoman rule, and consists of narrow streets and congested construction.

\section{DATA ACQUISITION AND PROCESSING}

\subsection{Spatial Data}

Spatial data has been collected so that all the necessary information could be drawn about the buildings in the area of Plaka and the study of its geometric evolution over the centuries. Four types of spatial data can be distinguished which were collected for different purposes. These include historical and modern maps, raster data and direct field measurements. The historical maps that were used derived from the cartographic and representational material found in [8] and constitute the product of long-term study and analysis of historical sources. They are a valuable resource for twelve chronological periods from the late Neolithic Age to the 20th century. Through these maps the user can observe the geometric changes in the area of Plaka over the centuries by overlaying maps of various time periods. Furthermore, users are given the opportunity to gain direct information on the time of creation of these monuments and the expansion of Athens over the centuries. Contemporary digital vector maps derived from secondary procedures and were either used as background to the study area or as a means to retrieve qualitative information about the buildings owned by the Ministry of Culture. They consist of a topographic map of Plaka, at a scale of 1:2000 created in 2002; street layout of Plaka created in 1974, plans defining specific conditions and limits on building in Plaka created in 1987, land use diagram of Plaka created in 1993 and plans defining listed buildings in Plaka created in 1980, all at a scale of 1:1000; and detailed façade plans and ground plans of buildings owned by the Ministry of Culture. The basic background of the 
IS consists of an orthophotomap and a stereo-restitution drawing produced using aerial images of Plaka at a scale of 1:10000, taken in 2001. The outline, roofs and volumes of buildings and blocks have been derived from the latter. The rest of the plans were used to distinguish buildings owned by the Ministry of Culture from others as well as $\tau$ o retrieve further information about everyone of these buildings. In addition, necessary geometric information, e.g., control points, detailed information for a 3D reconstruction of the internals of the buildings, etc, have been collected by field surveys. Moreover, for the study of the urban development of Plaka over the decades, large-scale aerial photographs were collected for five chronological periods from 1929 to 2001 . Further stereo-photogrammetric processing can lead to the creation of three dimensional city models for various time periods so that the development of the urban network can be studied, and detailed information concerning the area, height and volume of buildings may be determined.

\subsection{Attributes}

Digital data were collected to provide descriptive data to the GIS so that information can be retrieved according to users' needs. Organizing and storage of data were accomplished through the use of the relational database structure in ArcInfo 9.3 (INFO), where data are shown in tabular form. For each entity the type of descriptive data was defined along with the primary key of each table. Apart from the information derived from the spatial maps, a database recording the properties of the Ministry of Culture in Plaka in Microsoft Office Access environment was also used. Since the objective of this research focuses mainly on buildings owned by the Ministry of Culture, their descriptive data when inserted into the system are sufficiently detailed compared with those of other entities which have been mainly used for the visualization of the wider environment. In particular the attributes of the buildings owned by the Ministry of Culture are the area, the address, the building factor, the qualification as a listed building, the description of the current situation, their ownership's transfer to other institutions, the existing use, etc. This information was derived from existing databases and in situ data collection.

\section{DEVELOPMENT OF A 3D IS}

The need to acquire three dimensional information in recent years is rapidly growing in many scientific fields such as urban planning, architecture and visualization of environment, etc. This growing need has led to the rapid technological evolution in the production of specific software packages that serve this purpose. Therefore, three-dimensional GIS have been developed over the last decade as processing systems for spatial and descriptive information [9]. These systems have an advantage over two-dimensional GIS due to their capability of visualization and navigation through the depicted model space. However, although these systems should include the same advanced management features and analysis with two-dimensional systems, yet they have not been developed to the same extent. Usually, users employ it primarily as a tool for visualization and navigation, rather than as a stand-alone software to address a problem [10]. The use of three-dimensional GIS contributes to the representation and management of three-dimensional data which is of great importance for the better understanding of the data location relative to its surroundings. For the purpose of this project, a 3D GIS was developed taking advantage of its capabilities in order to represent 3D city models. The creation of this system was facilitated by the development of a 2D GIS which includes the entities that will be represented in three dimensions. The system was created in ArcScene environment which is a 3D Analyst extension for ArcGIS 9.3 and is used to visualize, manage and analyze three-dimensional vector and raster data.

\subsection{Two Dimensional Information System}

The design of an information system consists of different stages which determine the overall objective of implementation, the data input into the database, relationships between them, their constraints and the logical and physical organization. These stages are the conceptual, logical and physical design of an information system [11]. At the stage of conceptual design, the necessary entities were determined that will be processed in the system along with their attributes. These are the buildings owned by the Ministry of Culture, other buildings in the area, archaeological sites, churches and blocks. More detailed descriptive data was introduced about the buildings owned by the Ministry of Culture in accordance with the main subject of study. At the stage of logical design, the method of data organization and the software used to develop the system were selected. The structure chosen for the database is the relational database structure and the data is displayed in tabular form. Each entity has its own table where descriptive data is stored. The software used was the ArcGIS Desktop 9.3 and in particular the ArcInfo version. Firstly, the vector (topographic map) and raster data was inserted into ArcMap in order to form the background of the system. Then the types of data and their attributes were inserted into the built-in ArcGIS database (INFO). At the final stage of the physical design, details concerning data storage and management procedures were determined in order to produce the final products (Figure 1). After the database design various procedures were implemented concerning the data management and information retrieval. Such were the creation of spatial queries and the connection to external image, multimedia or CAD files. 


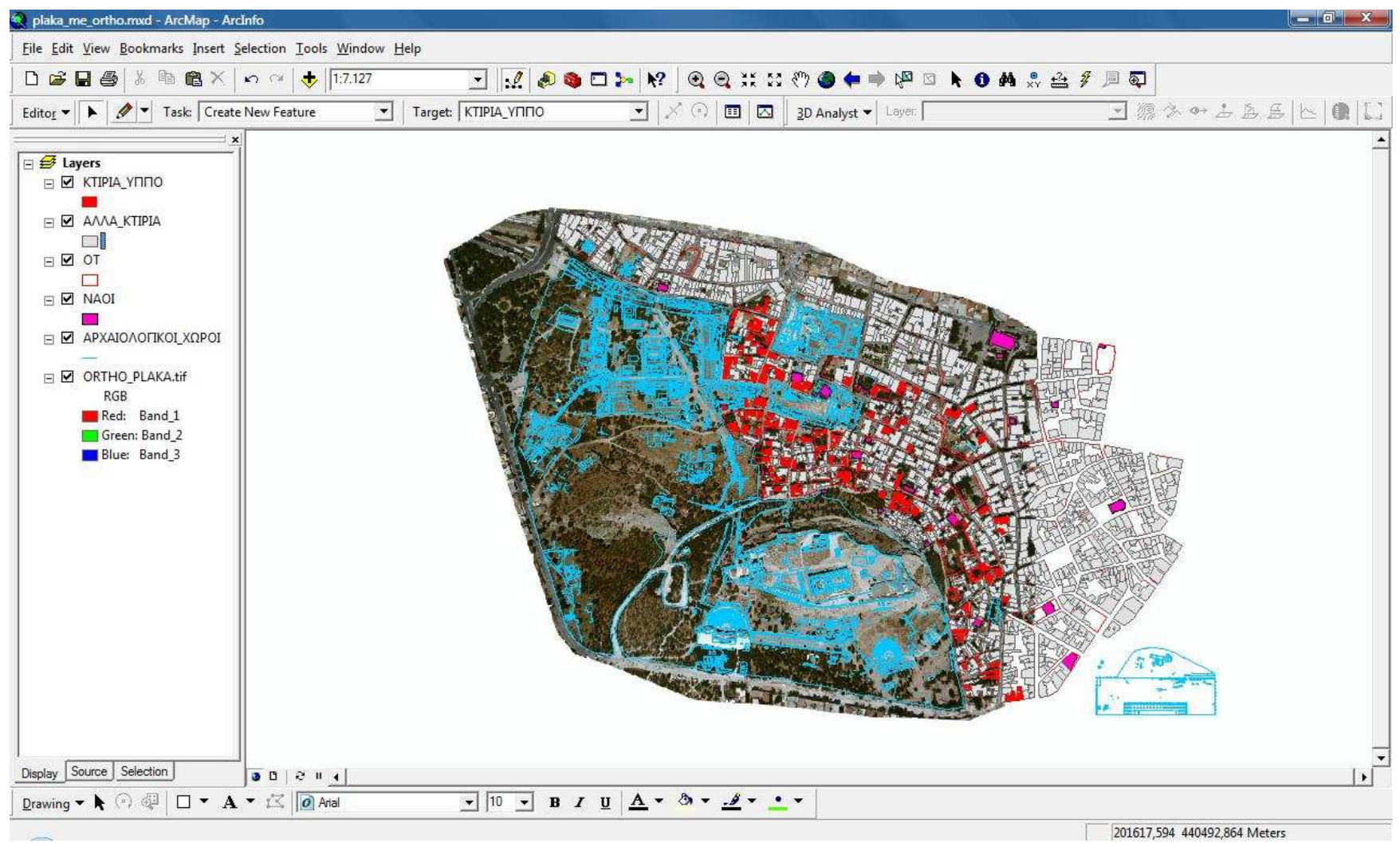

Figure 1: Screen showing vector and raster spatial data of the Geographic Information System

\subsection{Three Dimensional Information System}

The implementation of the 3D IS was held in ArcScene environment where the whole process was divided into two information levels, that of the background and of the buildings. For the creation of the background, a digital elevation model (DEM) and an orthophoto of Plaka were used. The DEM was created through the 3D Analyst toolbar using digitized contours of the study area, while the final background layer resulted from the process of draping the orthophoto on the surface (Figure 2 left). The next step consisted of the creation of 3D buildings which can be displayed at different levels of detail (LoD) depending on the users' needs. One of the reasons for the representation of buildings at different levels of detail is the system optimization relating to hardware limitations in a GIS environment. For instance, buildings depicted at a lower level of detail usually lead to a more effective management in 3D space. It is therefore obvious that due to computational power, the buildings depicted at higher level of detail refer to small areas compared to buildings of lower detail that can cover larger areas [12].
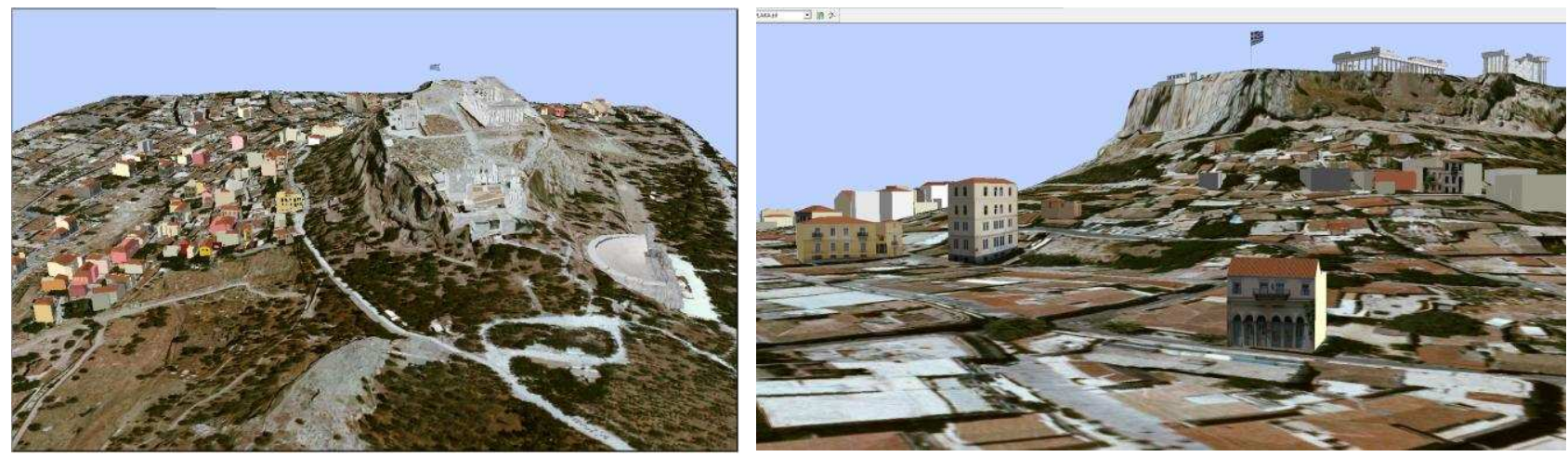

Figure 2: Left: Creation of the 3D background which resulted from a draping process. Right: Representation of buildings at the first level of detail (LoD). 
The first and lowest level of detail consists of the two-dimensional depiction of the buildings. This level in which there is no concept of altitude, meaning the volume of the building, shows the outlines of buildings as seen on topographic maps or ground plans (Figure 2 right). The depiction of the buildings at this level is the basis for the three-dimensional representations. At the second level of detail the buildings are represented by a plain volume without emphasis on building facades, roofs, etc. At this level of detail which was a plain coloring of the facades, buildings that are not owned by the Ministry of Culture were represented. The planimetric information came from the topographic map of the area while the vertical one resulted from photogrammetric measurements or on-site registration of floors of the buildings (Figure 3 left).
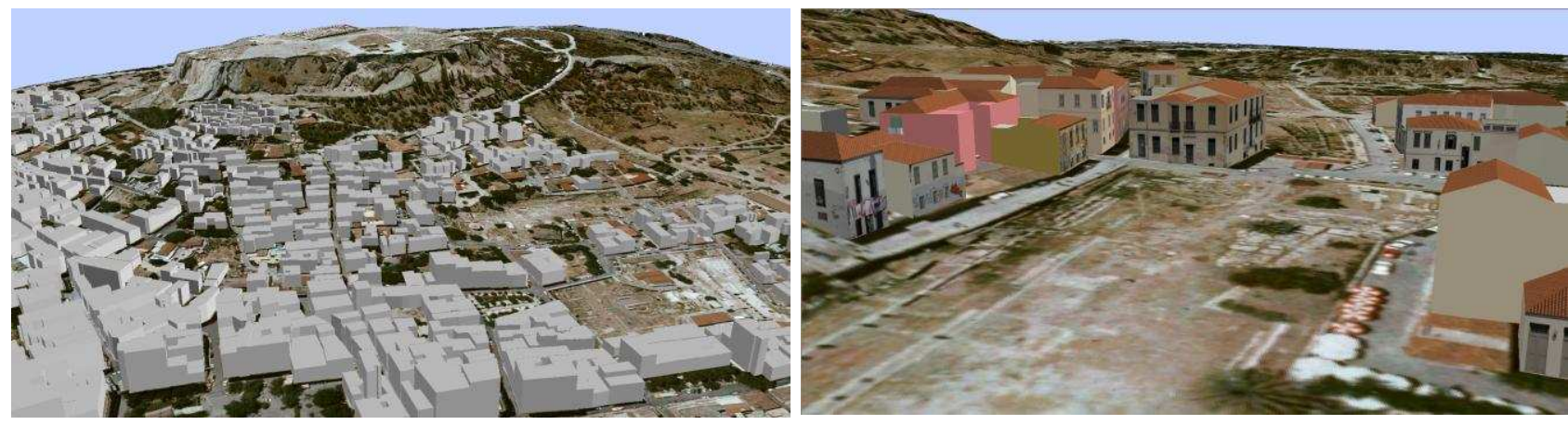

Figure 3: 3D representation of buildings at the second LoD (left) and at the third LoD (right) in ArcScene.

At the third level of detail, the buildings are depicted in more detail as their facades result either from unrefined images or orthophotos depending on users' requirements. The roofs of buildings are modeled in order that their geometry can be represented to a greater extent. Most buildings owned by the Ministry of Culture were represented at this level (Figure 3 right). In order to determine their height and volume 3D geometric information was collected from the stereo-photogrammetric processing of aerial images that were used to produce the orthophoto of Plaka. For the texture mapping of the facades of buildings, terrestrial photographs were taken. Specifically, for the buildings requiring more than one photo, geometric projections were carried out on individual photos using the rectification software Archis so that a photomosaic could be generated. Furthermore, by using the Photoshop CS3 software the noise and obstacles appearing in the photos were removed (Figure 4).

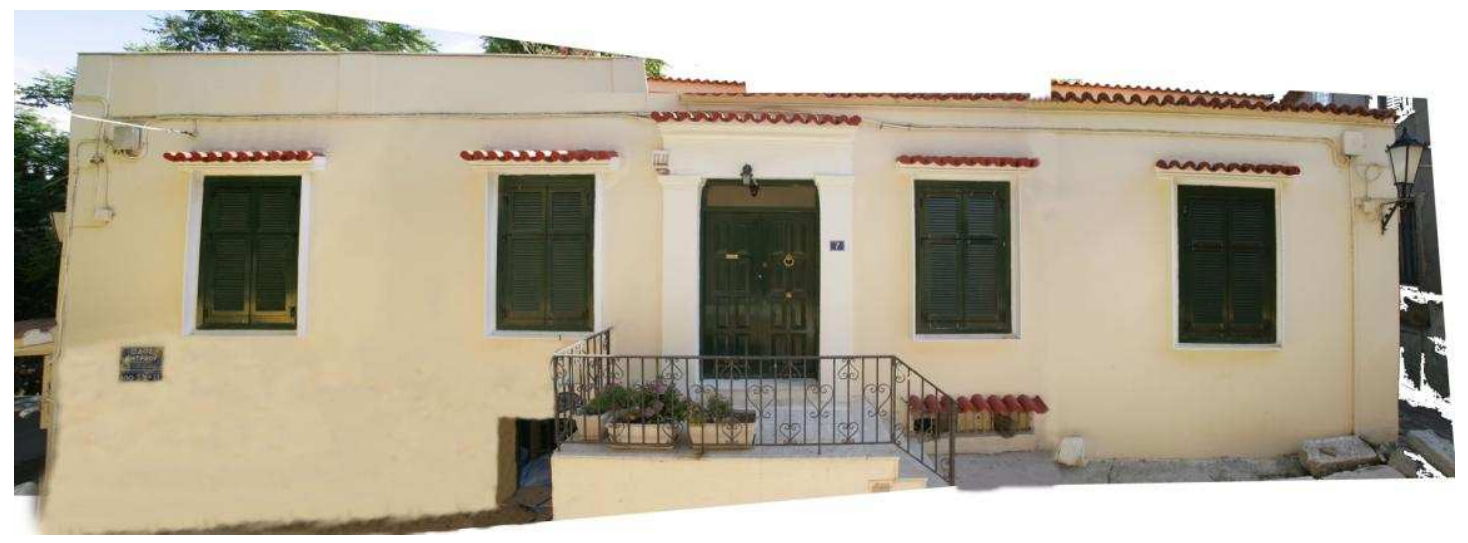

Figure 4: Photomosaic of a building's façade after editing. 
The next step regarded the creation of the 3D building models using the software Google SketchUp 6. This software was selected because of its compatibility with ArcScene to transfer models through a plug-in in the format of Microsoft Access (.mdb); the files were imported into ArcScene as multipatch features and not as symbols so that they can be managed and analyzed in 3D GIS. After importing the outlines of buildings, and on this basis along with the 3D geometric information, the volumes and roofs were modeled. At the fourth and highest level of detail, buildings are depicted in great detail since their facades and roofs are shown in their real form and not entirely through draping images. The more detail the building shows, the more geometric information is inserted in the IS which contributes to a more realistic representation. In some cases the buildings owned by the Ministry of Culture were represented at this level, with varying levels of accuracy. The accuracy of the desired scale sets the ground rules for collecting raw data. Such a model was created in 3D Studio Max and resulted from a combination of geodetic, photogrammetric and topometric methods for a 3D reconstruction at a scale of 1:50. Figure 5 illustrates views of the 3D model in 3D Studio Max (Figure 5).
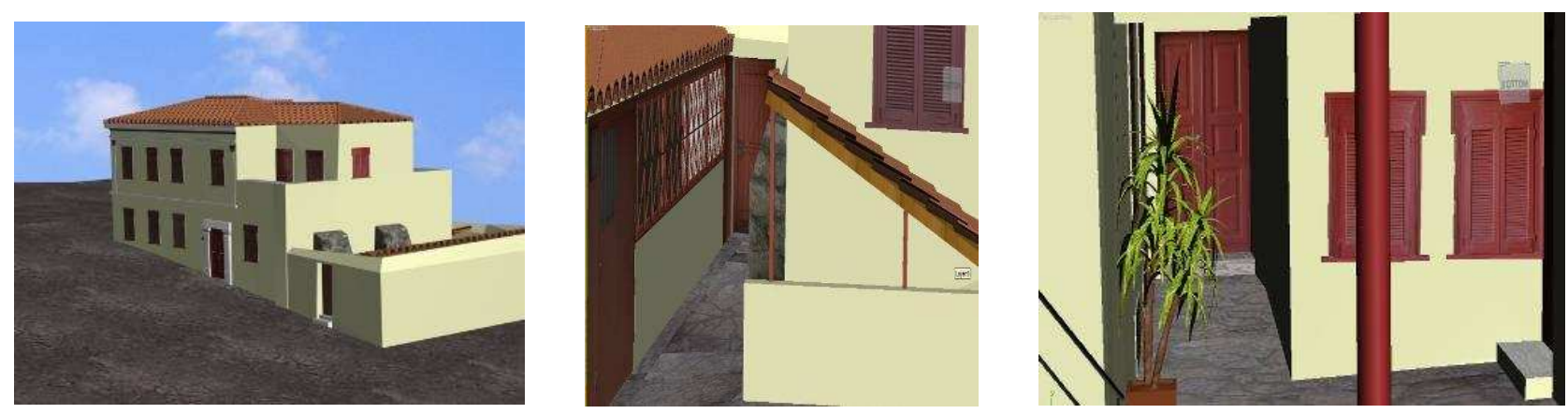

Figure 5: Perspective views of a 3D model building represented at the forth LOD, in 3D Studio Max

After the reconstruction of $3 \mathrm{D}$ building models, their attributes were imported in the database and linked to useful external files. Since the contents of the 3D GIS are common to the ones of the 2D GIS, the procedures performed to establish the two-dimensional system formed the basis for the three-dimensional one. Specifically, the common data was imported to the three-dimensional system by joining the tables of entities between the two systems.

\section{PRODUCTS OF THE INFORMATION SYSTEM}

\subsection{Two Dimensional Products}

The retrieval of qualitative and quantitative information in two-dimensional IS can be accomplished either by selecting specific geospatial data or by defining queries based on criteria. In the first method, ArcMap enables the user, through descriptive data of the database, to select any spatial element (e.g. building) using the tool Identify, thus obtaining information about the building directly, without having to engage with the database (e.g., through tables of attributes). Another advantage of the system is that it gives the user the opportunity to interact with external files, such as image, multimedia or CAD files, by selecting spatial data. In particular, the linkage of some buildings owned by the Ministry of Culture to external files, existing architectural plans, e.g., was implemented using the HTML popup tool. This tool displays an HTML page of the selected building containing a list of external data depending on the needs of the user. Such files may be textual information, old and new photographs, ground plans, views and sections as well as videos depicting the buildings to a higher level of detail (Figure 6 left), which is important in 3D IS in terms of volume. Another method for information retrieval in a two-dimensional system consists of the selection of spatial data based on attributes and location criteria. In this application the entities concerning the buildings owned by the Ministry of Culture were selected based on their attributes. For this purpose, several queries were formed, whose results were stored in separate thematic layers for the users' convenience. The queries were expressed through SQL, including the entities and their descriptive data.

\subsection{Three Dimensional Products}

The extraction of information mentioned above can also be applied to the 3D GIS by simply connecting it to the 2D IS. By linking tables between the two systems, layers of the three-dimensional system acquire both descriptive information of the features and qualitative information generated by creating links with HTML popup tool. In this way it is possible 
to combine the visualization of buildings with the database of the system, so as to extract the relevant information according to users' needs. Figure 6-right illustrates the corresponding example of connecting to external files in threedimensional level. Apart from the thematic information, ArcScene enables the user to create a three dimensional virtual tour video, showing all the building models and their surroundings in real time in order to allow navigation in 3D space. In this application, a series of images (keyframes) were captured, which were given a field of view (camera position) at specific intervals of the total length of the video. Afterwards, the generated video was exported to .avi format, in order to be compatible with most video player software, while the frame rate was set to 30 frames per second. In order to create a functional application, available computing power must be taken into account, since depending on the number of buildings depicted (which in our study were 132) and their detailed representation, a very powerful computing system may be required. For this reason the 3D building models of lower levels of detail were linked to videos of buildings represented in the highest level of detail and accuracy. Such a system becomes more convenient for users while it does not require time-consuming procedures for either the update of existing data, insertion of additional data or even information retrieval in three-dimensional level.
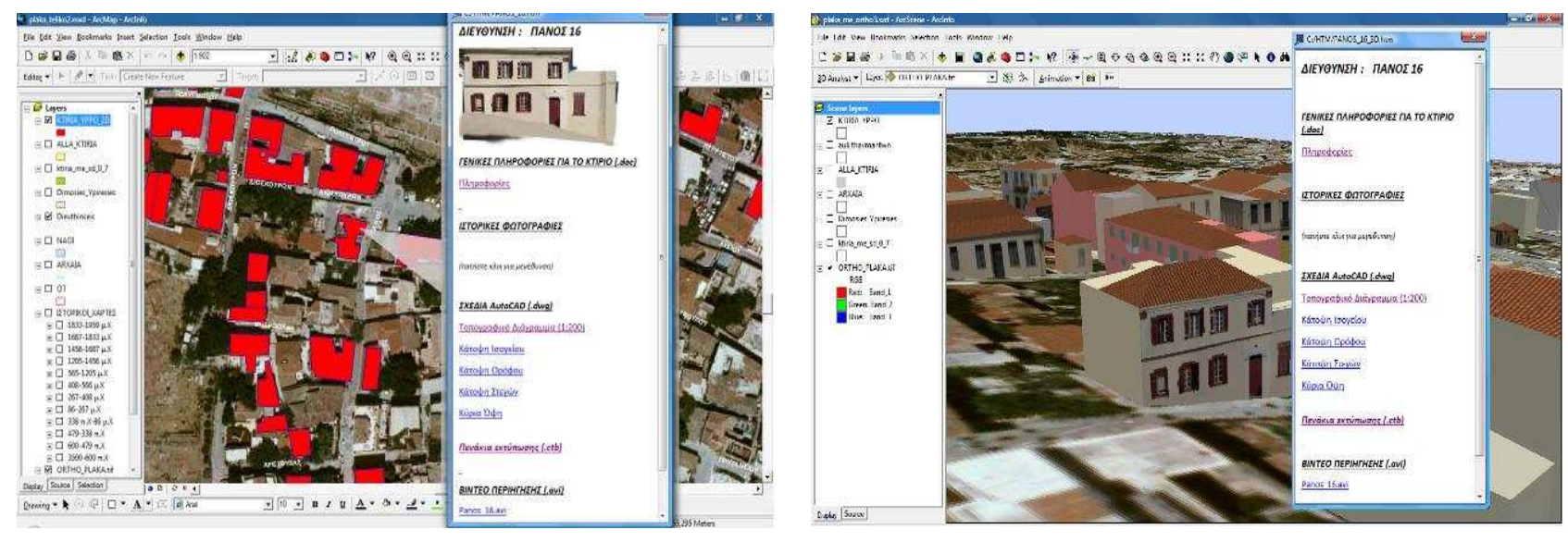

Figure 6: Linkage of buildings to external files via HTML popup tool in 2D (left) and 3D space (right).

\section{CONCLUSIONS}

The old city centre of Athens is a unique and very interesting area. It combines very important ancient, roman and byzantine monuments in the urban area strictly controlled by limitations in building constructions and land use. Thus, it consists of small blocks with low-height buildings (up to 3 floors) and very narrow streets creating a complex urban fabric. The aim of this project was not to create another 3D city model, similar to what has been done for many historical European cities. The main objective was to create a detailed 3D Information System for more than 100 buildings owned by the Ministry of Culture, which are scattered throughout the area. Thus an IS was developed that combines descriptive information about these buildings, e.g., use, legal status, images, drawings, etc, with the spatial information in three dimensions, with simultaneous geometric documentation and with display of their shell and the surrounding area. In the interest of a complete and proper presentation, the other buildings in the area were represented as 3D models in simpler ways and forms including descriptive data in the database. Such a system gives the capability of managing and analyzing large volumes of data that can be linked with external files and constantly updated. This study combines digital photogrammetric techniques with GIS. Photogrammetry is an efficient, alternative solution to traditional methods of measurement and provides several economic techniques for the acquisition of three-dimensional data. By using digital photogrammetric techniques one can take the appropriate measurements of objects on images, considerably reducing the amount of field work. The three-dimensional products can serve many purposes since the IS is a tool for reference, measurement and representation of buildings in three dimensions. In this particular case, the system provides the opportunity of optimal management of properties owned by the Ministry of Culture, while monitoring the current situation so as to take appropriate measures to safeguard the cultural heritage of buildings or intervene at any economic or architectural - building level. Further study will be carried out about 3D representation of the Plaka area at different periods, so that there is a comprehensive view of changes every few decades while giving the opportunity to manage and enrich the system depending on each project. 


\section{REFERENCES}

[1] Wang, L., Hua, W.: Survey and Practice of 3D City Modelling, Lecture Notes in Computer Science, Berlin, 2006, vol. 3942, pp. 818-828.

[2] Benoit,F., Alain,L.: 3D City GIS - A Major Step Towards Sustainable Infrastructure, http://ftp2.bentley.com/dist/collateral/whitepaper/WP_3D_City_GIS_long.pdf, 2011-05-11.

[3] Zlatanova, S., Rahman, A., Pilouk, M., 2002. Trends in 3D GIS Development, Journal of Geospatial Engineering, Hong Kong, 2002, vol. 4, No. 2, pp. 71-80.

[4] Kirimura, T., Yano, K., Kawaguchi, H.: Applicability of 3D GIS to the view preservation policy of Kyoto City, Proceedings of 22nd CIPA International Symposium, Kyoto, Japan, 2009.

[5] Gandolfi, S., Barbarella, M., Ronci, E., Burchi, A.: Close photogrammetry and laser scanning using a mobile mapping system for the high detailed survey of a high density urban area, Proceedings of The International Archives of the Photogrammetry, Remote Sensing and Spatial Information Sciences, Beijing, China, 2008, vol. XXXVII, part B5, pp. 909-914.

[6] Tunc, E., Karsli, F., Ayhan, E.: 3D city reconstruction by different technologies to manage and reorganize the current situation, Trabzon, Turkey, 2004,

www.isprs.org/proceedings/XXXV/congress/comm4/papers/388.pdf, 2011-05-11.

[7] Malumpong, C., Chen, X.: Interoperable three-dimensional GIS city modeling with geo-informatics techniques and 3D modeling software, , Proceedings of The International Archives of the Photogrammetry, Remote Sensing and Spatial Information Sciences, Beijing, China, 2008, vol. XXXVII, part B2, pp. 975-979.

[8] Travlos, J: Urbanization of Athens - From the pre-historic years until the beging of the $19^{\text {th }}$ century, KAPON Editions, Athens, ISBN: 960-7254-01-5,1993 (in Greek).

[9] Abdul-Rahman, A., Pilouk, M.: Spatial Data Modelling for 3D GIS, Springer-Verlag Berlin Heidelberg, Berlin, 2007.

[10] Stoter, J., Zlatanova, S.: 3D GIS, where are we standing, http://repository.tudelft.nl/assets/ uuid:baa06f95-bb9443b1-8854-174f4259c17d/gdmc_stoter_2003d.pdf, 2011-05-11.

[11] Longley, P., Goodchild, M., Maguire, D., Rhind, D.: Geographical Information Systems and Science, John Wiley \& Sons Ltd, U.K., 2005.

[12] Kolbe, T. H., Gröger G.: Towards unified 3D city models, Institute of Cartography and Geoinformation, University of Bonn, Germany, 2003, www.ikg.uni-bonn.de/fileadmin/sig3d/pdf/cgiav2003_kolbe_groeger.pdf, 2011-05-11. 